\section{Heterogeneity of Developmental Dyscalculia: Cases with Different Deficit Profiles}

\section{Abstract}

Context:_The aim was to further understand the heterogeneity of developmental dyscalculia (DD). Utilizing four children (8-9 year-old) performance was contrasted against predominant hypotheses of DD.

Case report: Despite showing similar mathematical deficits, these children showed remarkable inter-individual variability regarding cognitive profile and deficits. Two cases were consistent with the approximate number system deficit account, and the general magnitude-processing deficit account. One case had an access deficit in combination with a general cognitive deficit. One cases suffered from general cognitive deficits only.

Conclusion: The results showed that DD cannot be attributed to a single explanatory factor. These findings support a multiple deficits account of DD and suggest that some cases have multiple deficits, whereas other cases have a single deficit. We discuss a previously proposed distinction between primary DD and secondary DD, and suggest hypotheses of dysfunctional neurocognitive correlates responsible for the displayed deficits.

Keywords: Developmental dyscalculia; Symbolic number processing; Nonsymbolic number processing; Time processing; Spatial processing

Received: December 09, 2015; Accepted: January 21, 2016; Published: January 29, 2016

\section{Introduction}

Increasingly more attention is being directed towards identifying the neurocognitive profile and origins of developmental dyscalculia (DD), a specific learning disorder characterized by severe impairments in acquiring mathematical competency [1]. Important strides have been made and different single core deficit hypotheses have been proposed. Each hypothesis has received some empirical support from both behavioral and neuroimaging studies.

It has been known for quite some time that children show different kinds of mathematical difficulties [2]. Thus, it is increasingly recognized that $\mathrm{DD}$ is heterogeneous and the observed phenotype(s) might be caused by a multitude of underlying neurocognitive causal factors [3-5]. Determining these specific causal factors is further complicated by frequent comorbidities, such as ADHD or dyslexia [6]. Consequently, some researchers propose that the scientific community should differentiate between primary and secondary DD [3, 7]. Primary DD is characterized by a severe deficit in numerical or arithmetic

\section{Ulf Träff, Linda Olsson, Rickard Östergren and Kenny Skagerlund}

\author{
Department of Behavioural Sciences and \\ Learning, Linköping University, Campus \\ Valla, Linköping, Sweden
}

Corresponding author: Kenny Skagerlund

Department of Behavioural Sciences and Learning, Linköping University, Campus Valla, SE-581 83 Linköping, Sweden

\section{Đ Kenny.skagerlund@liu.se}

Tel: +4613282103

Fax: +4613149403

Citation: Träff U, Olsson L, Östergren R, et al. Heterogeneity of Developmental Dyscalculia: Cases with Different Deficit Profiles. J Child Dev Disord. 2016, 2:1. functioning, caused by different underlying biological factors. Secondary DD denotes individuals whose impaired numerical capacity can be explained entirely by non-numerical impairments, such as attention or working-memory processes [3]. Thus, even primary DD may be subject to further subtyping, dependent on different underlying factors.

In order to implement appropriate educational interventions, it becomes crucial to identify the subtypes and their underlying causes. Therefore, not only behavioral measures such as test scores, error rates and response times should be used to describe DD. To identify the neural microstructures and genetic dispositions causing the observed phenotypes, several levels of descriptions should be used, thereby creating a multilevel taxonomy, which can also be used to guide further neuroimaging studies.

In the current study, four children with homogenous mathematical-behavioral profiles fitting the characteristics of DD were selected to provide a clear demonstration of the heterogeneity of DD. The purpose was to investigate the cognitive and number processing skills of the four children and relate their 
behavioral profile to predominate hypotheses. To this end, three levels of description were used: (1) At the behavioural level, four children were identified suffering from DD (2) at the cognitive level, a comprehensive test battery was administered to evaluate the cognitive profile and performance, (3) at the neurocognitive level, the results of the above mentioned levels were used to hypothesize about the underlying neurocognitive correlates with the purpose of guiding future neuroimaging studies. Thus, the candidate neural structures can be used, for example, in RegionOf-Interest (ROI) analyses to investigate their role in DD.

\section{Core hypotheses regarding DD}

A predominant hypothesis is that DD originates from a core deficit in the innate Approximate Number System (ANS), which enables humans to represent quantities in an approximate manner. It has been suggested that this system constitutes the foundation onto which the symbolic number system is mapped [8-10]. By having participants determine which of two simultaneously - and briefly - presented sets of objects is more numerous, individual ANS acuity can be measured. Using psychophysical modelling, given the assumption that the ANS operates according to Weber's law, individual Weber fractions (w) can be used as an index of individual ANS acuity [11]. ANS acuity has been found to be related to mathematical proficiency $[12,13]$ and studies have found that children with DD have poorer ANS acuity than their typically achieving peers $[5,11,14-16]$.

Butterworth and colleagues $[17,18]$ present a second core deficit account of DD, the numerosity-coding hypothesis. It posits that $D D$ is due to a deficit in the internal number code that represents each quantity exactly as a set of discrete elements within and above the small number range. Unlike the conceptualization of the ANS, the numerosity coding hypothesis maintain that human beings are equipped with a number system that can represent larger sets in an exact manner, much like the OTS (see below) for smaller numbers. Support for the notion that children with DD have problems with non-symbolic and exact enumeration has been found in a study on Chinese children [19].

Neuroimaging studies have begun to map the neurocognitive correlates of number processing and arithmetic. Research indicates involvement of the intraparietal sulcus (IPS) during nonsymbolic number processing (e.g. during ANS tasks) and arithmetic calculations [20]. Furthermore, children with DD have both functional and structural abnormalities in this region in terms of gray matter volume and activation patterns [21-23]. Further support for the involvement and importance of the IPS comes from a recent study by luculano and Cohen Kadosh [24]. By using transcranial stimulation of the posterior parietal cortex individuals diagnosed with DD showed improved numerical proficiency. This highlights the role of the parietal cortex in number processing and, provides promising prospects of effective interventions [24].

Recent studies indicate that children with DD or mathematical difficulties not only have number processing deficits but also problems in processing other magnitudes such as time and space [25-28]. For example, Skagerlund and Träff [27] found that 10 -years olds with DD showed impaired ANS acuity (i.e., non-symbolic number discrimination), but also problems with time discrimination and difficulties with two spatial skills; spatial visualization (paper-folding) and mental rotation. The results support the "A Theory Of Magnitude" (ATOM) model [29, 30], which states that time, space and number are processed by a partly shared general magnitude system. However, each dimension is also supported by dimension-specific processess [31-33]. Strong evidence for a shared magnitude system is that time and space display the typical effects of distance, size and SNARC (Spatial Numerical Association of Response Codes) effect consistently found for numbers [33]. The distance effect refers to the fact that the selection of the larger of two numerals is performed faster when the numerical distance large ( 1 vs. 9) is compared to when it is small (1 vs. 2) [34]. The fact that comparing numbers becomes increasingly difficult the larger they are, even when the distance between them is kept constant (e.g., comparing 8 and 9 is more difficult than comparing 2 and 3) constitutes the classical problem-size effect. The SNARC effect entails faster left-sided responses to smaller numbers and faster right sided responses to larger numbers. These three effects are considered to demonstrate that numbers are spatially represented as approximate analogue magnitudes (i.e., mental number line) in an ascending left to right order, that are automatically accessed when numerical information is processed. The distance effect has been shown for many different non-numerical magnitude stimuli such as area [35], length, time [36], and pitch [37]. The size effect has also been observed with non-numerical magnitude stimuli, for example, Fias, Lammertyn, Reynvoet, Dupont and Orban, [38], obtained a size effect when subjects compared pairs of angles or pairs of lines. The same is true for the SNARC effect, for example, Ishihara, Keller, Rossetti, and Prinz [39, see also 27] demonstrated that time is also spatially represented, resulting in the so-called STEARC effect (Spatial-Temporal Association of Response Codes). Another line of evidence for a shared magnitude system has been provided by experimental studies examining the interaction between magnitudes. A number of studies demonstrate bidirectional influence between space and number [38, 40]. Similar influence has been found between number and time, but with time processing more often affected by number processing than vice versa [41]. Also space and time have demonstrated to exert reciprocal influence on each other [39, 42].

Skagerlund and Träff's results [27] were also in accordance with Feigenson [43], who suggested that if the diverse magnitude representations share a common mechanism, deficits in one dimension should be paralleled by deficits in other magnitude processing abilities. This led Skagerlund and Träff [27] to suggest that children with $\mathrm{DD}$, whose primary deficit was thought to be circumscribed to the ANS prior to the study, may in fact suffer from a more comprehensive magnitude processing deficit that extends from quantity processing to also include processing of time and space.

Humans are also believed to be equipped with a second system involved in number processing, called the object-tracking system (OTS) [10, 16, 44, 45], a visuospatial object-based attention system for keeping track of 3-4 objects exactly. One characteristic of the OTS is that it allows effortless and quick apprehension of 1-4 objects, called subitzing. Earlier research indicates that 
children with DD have a restricted subitizing range of a maximum of 3 objects [44, 46-48], whereas typically developing children can quickly apprehend up to 4 objects [49]. The OTS is dissociated from both the ANS and the numerosity-code in that the OTS operates on individual objects and is based on visuospatial attention, whereas the ANS and numerosity-code are dedicated systems pertaining to numerosity alone [18].

The access deficit hypothesis [50] states that DD is caused by a defective connection between the symbols (e.g., digits) and the underlying magnitude representations. Thus, DD is not due to a deficit in the innate ANS per se $[45,50]$. Evidence has been reported by De Smedt and Gilmore [51], Landerl and Kölle [52], and Rousselle and Noël [50] as children with DD displayed problems with symbolic number comparison, but normal performance on non-symbolic number comparison. Although Skagerlund and Träff [5] found a subgroup of children with DD showed poor ANS acuity, another subgroup was also identified. They showed intact ANS', but had difficulties in accessing the underlying semantic representation. This led the authors to suggest that DD is heterogeneous disability with different subtypes, including one characterized by an access deficit [5]. The access deficit hypothesis has also received support from neuroimaging studies using diffusion tensor imaging (DTI) to explore white matter integrity in the brain in individuals with and without DD. White matter development is an important aspect of brain maturation, reflecting connectivity between brain areas, and it is associated with learning [53]. For example, Rykhlevskaia, Uddin, Kondos, and Menon [54] found that their participants exhibited abnormal right hemisphere temporal-parietal white matter. A network analysis led the researchers to suggest that DD is a disconnection syndrome [55]. Another study focused on the developmental trajectory of grey and white matter, where DD children did not show increases in white matter in the frontal lobes nor in the parietal lobes as a function of age, which could be observed in controls [53]. This may suggest that the frontal lobes do not connect adequately to the parietal lobes during ontogenetic development. The parietal lobes consist of key structures, such as the IPS and the angular gyrus (AG). The AG is believed to be involved during retrieval of arithmetical facts and semantic processing of numerical symbols [53]. These neuroimaging studies provide support for the notion that DD is, at least partly, a disconnection syndrome. These individuals with DD may struggle with the retrieval of arithmetical facts and accessing the non-symbolic magnitude representations even if the representations themselves, and the cortical loci subserving them, are intact.

The general cognitive deficit hypothesis is a fundamentally different account of DD, postulating that deficits in the underlying general cognitive system impede the development of ageadequate mathematical skills $[2,56]$. Numerous studies have found that children with DD display general cognitive deficits, for example, working memory, executive functions, processing speed [57-64]. These findings in the view of primary and secondary DD, suggest that these children have secondary $\operatorname{DD}[3,7]$.

To summarize, it is clear from previous studies that have used group comparisons that the origin of DD is multifactorial and not solely caused by a core deficit [4, 45, 65-68]. In fact, Andersson and Östergren [46] obtained data consistent with three core deficit accounts (defective ANS; ANS and OTS; general cognitive deficits) in one sample of children. The results are in favor of the multiple deficits account rather than a core deficit account.

\section{Developmental dyscalculia as a heterogeneous neurocognitive disorder}

The multiple deficits account is reasonable considering that prior studies have all used group comparisons. A flaw of this research method is the lack of sensitivity to the variability among individuals with DD; although no significant group differences may emerge on a particular task, some DD participants still display severe difficulties. Conversely, some DD participants may display adequate performance on tasks that show significant group differences. Thus, only relying on traditional group comparisons might not be the optimal design for exploring the heterogeneity of a phenomenon such as DD. Collapsing all individuals into a single, supposedly, homogeneous group may obfuscate important individual variability. In this paper, four cases of DD are analyzed to further understand the heterogeneity regarding the origin(s) of DD.

Although the bulk of research on DD has ignored its heterogeneity, there are some recent exceptions. Bartelet, Ansari, Vaessen and Blomert [69] examined a sample of 226 children (grade 3-6) with DD on a comprehensive test battery of number processing and general cognitive tasks and used cluster analysis to identify subgroups of DD with different cognitive profiles. The cluster analysis generated six clusters; Cluster 1 was characterized by problems with the number line estimation task but no other problems. Cluster 2 was characterized by problems with the approximate numerical knowledge and number line estimation tasks. Cluster 3 displayed the same problems as cluster 2 but also weak spatial short-term working memory. Cluster 4 was distinguished by weak symbolic number processing. Cluster 5 did not display any number-specific cognitive processing weaknesses, additionally having a strong verbal short-term working memory. Cluster 6 was characterized by low nonverbal $I Q$, but no other problems. The clusters support the notion that $\mathrm{DD}$ is a heterogeneous disorder with a multifactorial origin.

In view of the core deficit hypotheses, cluster 2 and 3 correspond to the defective ANS hypothesis, whereas cluster 4 is consistent with the access deficit hypothesis. Cluster 1 demonstrates that some children with DD have specific problems in developing an accurate symbolic number line, which can be considered a higher level of number processing (cf. von Aster and Shalev) [70]. In contrast, none of the clusters were in line with the hypothesis of general cognitive deficits. Furthermore, cluster 5 suggests (still) unexplored origins of DD. A tentative interpretation is that the arithmetic impairment in this subgroup can be attributed to exogenous factors, such as poor motivation or education [69].

Further support for the multiple cognitive deficits hypothesis is provided by Skagerlund and Träff [5], as they examined children with different profiles of mathematical deficits. They found that children with calculation and arithmetic fact retrieval problems suffered from an impairment of the ANS, whereas children with 
only arithmetic fact retrieval problems suffered from an access deficit.

A few researchers have used case studies to examine the heterogeneity of DD luculano et al. [71] studied two boys with DD. Neither case demonstrated problems with non-symbolic approximate number processing, excluding the possibility of a defective ANS. The first case's results were consistent with the access deficit hypothesis, as performance was poor on the symbolic number comparison task. The second case displayed weak dot enumeration performance, but performed at normal levels on all other number tasks. This performance pattern suggested a deficit with processing exact numerosities, in accordance with the defective numerosity-coding hypothesis $[17,18]$.

Another case study was DB, a 42-43 year old woman with good overall cognitive capacities but who suffered from a severe deficit in arithmetic fact retrieval [72]. DB's results suggested an intact ANS as well as intact access to it from symbols. However, extensive testing of her long-term memory indicated a hypersensitivity-tointerference, preventing DB from establishing an arithmetic facts network.

In sum, prior studies reveals that three different lines of research provide convergent evidence that DD originates from multiple deficits: traditional studies making group comparisons by testing and contrasting different core deficit accounts, studies aiming at identifying subgroups with different deficit profiles by making group comparisons, and case studies aiming at identifying the full spectrum of heterogeneity regarding the origin(s) of DD.

\section{The present study}

The aim of the present paper was to expand our knowledge regarding the origin(s) of $\mathrm{DD}$, by testing the main core deficit accounts previously described in relation to four cases of DD with similar profiles of mathematical deficits. The ATOM hypothesis, that a general magnitude-processing deficit underlies DD, was also tested.

The main research question was whether all four cases displayed similar cognitive deficit profiles consistent with the same or different core deficit accounts. The latter outcome would suggest that DD originates from multiple deficits. The cognitive profile of each child depends on the performance patterns on the tasks that measure the abilities that relate to the aforementioned hypotheses of DD. For example, if a child shows poor ANS acuity, poor spatial processing and poor temporal processing, but within normal range on the other measures, we would conclude that this child suffers from primary DD with a magnitude processing subtype. By contrast, if a child shows intact domain-general abilities and intact magnitude processing but poor symbolic number processing, as measured using a symbolic number discrimination task, we would conclude that this child may have primary DD with an access-deficit subtype. The label secondary DD would be befitting, for example, if a child shows intact number processing abilities across the board, but show impaired workingmemory ability.

\section{Materials and Method}

\section{Case descriptions}

The four cases consist of three second-graders and one thirdgrader. The four cases are termed C1 (boy, 8 years, 4 months), C2 (girl, 9 years, 5 months), C3 (girl, 8 years, 7 months), and C4 (boy, 8 years, 2 months). Swedish was their native language, and they had normal or corrected-to-normal visual acuity and no hearing loss. The four cases were selected based on four criteria: 1) The child should have received special education in mathematics at the time of and at least one semester prior to the study. 2) The child's score on three of the five arithmetic measures (see below) had to be at or below the $10^{\text {th }}$ percentile (i.e., at or above z-score of -1.29) of the norms. 3) The child should not have any history of neurologically based impairments, such as ADHD or other known learning disabilities (e.g., dyslexia), neither were they subject to assessment of ADHD or other learning disabilities. 4) To exclude poor schooling and general intellectual impairments as underlying causes of low mathematical performance, the child's score on standardized reading tests and Raven's Standard Progressive Matrices (see below) had to be above the $15^{\text {th }}$ percentile (i.e., above z-score of -1.00) of the norms. The for cases' raw scores (z-scores) on Raven's, reading, and arithmetic tasks, along with mean performance (SD) for the norm groups are presented in Table 1.

All cases performed within the normal range on Raven's, the two reading tasks, as their $z$-scores were above -1.00 . None of them had circumscribed problems with a specific mathematical skill, such as arithmetical fact retrieval, a proposed subtype of DD [5, 72], but rather show pervasive mathematical difficulties across several aspects of mathematics.

Table 1 Performance (z-scores) of the four cases of DD and mean performance (SD) of the norm groups on Raven's, reading and arithmetic.

\begin{tabular}{|c|c|c|c|c|c|c|}
\hline & C1 & C2 & C3 & C4 & & \\
\hline Task & Boy Grade 2 & Girl Grade 3 & Girl Grade 2 & Boy Grade 2 & Norms Grade 3 & Norms Grade 2 \\
\hline Ravens & $17(-0.38)$ & $18(-0.98)$ & $18(-0.26)$ & $16(-0.51)$ & $24.06(6.11)$ & $20.17(8.24)$ \\
\hline Word-decoding & $106(0.77)$ & $88(-0.60)$ & $113(0.98)$ & $110(0.89)$ & $105(28.50)$ & $80.50(33.00)$ \\
\hline Reading comprehension & $6(-0.31)$ & $8(-0.48)$ & $7(-0.06)$ & $5(-0.56)$ & $10.22(4.26)$ & $7.24(3.98)$ \\
\hline Calculation & $0(-1.29)$ & $4(-0.55)$ & $0(-1.29)$ & $0(-1.29)$ & $5.36(2.49)$ & $2.96(2.29)$ \\
\hline Equation & $1(-1.12)$ & $0(-3.03)$ & $1(-1.12)$ & $0(-1.51)$ & $7.36(2.43)$ & $3.86(2.55)$ \\
\hline Arithmetic fluency & $3(-2.10)$ & $6(-1.94)$ & $11(-1.34)$ & $9(-1.53)$ & $32.18(13.48)$ & $25.18(10.58)$ \\
\hline Number facts (3 sec.) & $0(-0.94)$ & $0(-1.46)$ & $0(-0.94)$ & $0(-0.94)$ & 10.09 (6.93) & $5.62(5.99)$ \\
\hline Number facts (>3 sec) & $2(-11.48)$ & $17 *(-3.17)$ & $2(-11.48)$ & $20 *(-0.89)$ & $2.71(1.80)$ & $21.51(1.70)$ \\
\hline
\end{tabular}

* Finger counting 
The $\mathrm{C} 1$ case performed at/below the cut-off criterion of $z=-1.29$ on three (calculation; arithmetic fluency; number facts $>3 \mathrm{sec}$ ) of the five arithmetic measures. The $\mathrm{C} 2$ case performed below the cut-off criterion on all measures except for the calculation task. A distinctive feature of her skills is that she used a finger counting when solving simple single-digit arithmetic problems (number facts $>3 \mathrm{sec}$.). Despite this strategy, she scored considerably below the norm group mean on the number facts measure ( $>3 \mathrm{sec}$.). The $\mathrm{C} 3$ case displayed severe problems with the calculation task, the arithmetic fluency and the number facts (>3 sec.) measure. Her performance was also weak on the equation, and the number facts ( $<3 \mathrm{sec}$.) measures. The $\mathrm{C} 4$ case showed impaired performance on the calculation, the equation, and the arithmetic fluency tasks, whereas his performance on the number facts (>3 sec.) measure was normal. This distinction in performance is probably due to C4's use of finger counting when solving singledigit problems.

\section{Norm groups}

Three independent and unselected groups were used as norms for the case in third grade. The first norm group (equation and shifting task) included 53 children, the second norm group (visuospatial working memory, time discrimination, number naming) included 115 children, and the third norm group included $274-292$ children (all other tasks). Two independent and unselected groups were used as norms for the three cases in second grade. The sample sizes of the fourth norm group (reading comprehension, calculation, addition fluency, verbal working memory, color naming, mental rotation) included 292-303 and the fifth norm group (all other tasks) included 66 children. With respect to fluid intelligence, the groups showed scores within the normal range: norm group 1 ( $M d=56$ percentile, minimum=5, maximum=95), norm group 2 ( $\mathrm{Md}=59$ percentile, minimum=5, maximum=95), norm group 3 ( $M d=50$ percentile, minimum=1, maximum=98), and norm group 5 ( $M d=67$ percentile, minimum=15, maximum=95) displayed normal scores on fluid intelligence measured by Raven's Standard Progressive Matrices [73]. Norm group 4 displayed normal scale scores ( $M d=10$, minimum=4, maximum $=18$ ) on fluid intelligence measured by Wechsler's Matrix Reasoning test [74]. The test used to tap word-decoding was a standardized and normed test [75]. The norms were based on 145 third graders and 164 second graders. All children in the norm groups reported having Swedish as their native language, normal or corrected-to-normal visual acuity, and no hearing loss.

\section{Measures}

A comprehensive test battery was administered, tapping basic mathematical and reading skills, fluid intelligence (Raven's), and general cognitive abilities (e.g., working memory). Tasks tapping magnitude processing (number, temporal, spatial) were also included. The selection of tasks was based on current hypotheses regarding the origin of DD.

\section{Raven's standard progressive matrices}

This test of fluid intelligence is well known [73] and frequently used. It consists of a series of visual pattern designs with a piece missing, where the child selects which of six-eight options displayed beneath the design is the missing piece. The test includes five sets of designs (A, B, C, D, E), with 12 items per set. Only sets $B, C$ and $D$ were used in this study. The child received a test booklet, and after two demonstration/practice items had been performed with the experimenter, the child individually completed the 36 items at her/his own pace.

\section{Reading (word-decoding)}

In this standardized and normed test [75], the child read as many words as possible from a list of 100 words, presented in four columns with 25 words in each column, during 45 seconds. The child was instructed to read as quickly as possible without making any errors. The experimenter used a stopwatch to keep track of time, continually checked the child's answers and registered each error. The task consisted of two sheets of paper, A- and B-version, and the child performed both versions beginning with the A-version. The combined number of correctly read words from the two versions was used as the dependent measure.

\section{Reading comprehension\}

This test consisted of a short story read by the child [76]. The narrative took the form of a fairy-tale, and scattered throughout the text were single missing words replaced by a blank space and a bracket containing four words. The child then had to select which of the words made the most sense in terms of the sentence and the story, and underlined their answer. This reading test contained twenty items (i.e., missing words) scattered evenly throughout the story. The number of correct items selected within four minutes was the dependent measure.

\section{Arithmetic calculation}

Using three pen-and-paper tasks, calculation ability was tapped. The items were designed to become increasingly difficult. Number of correctly solved problems was the dependent measure. The same procedure (i.e., instructions, paper and pencil, scoring procedure) was used on all three subtasks. In the first calculation subtask, the child was asked to solve six addition and six subtraction problems (e.g., 57+42; 78-43; 568+421; 658437 ) in eight minutes. The problems were presented horizontally, and the child responded in writing. All problems, except two, involved regrouping (i.e. carrying or borrowing). The child could solve the problems in any way according to their own preference. However, only paper and pencil was at their disposal.

\section{Arithmetic equations}

The tasks consisted of 12 equations presented horizontally (e.g., $61+\ldots=73$; _ _ $+25=500$ ), where the child had to enter the correct digit so the equation was correct. The child was allowed seven minutes to perform the task.

\section{Arithmetic fluency}

The task was to solve as many single-digit addition (e.g., 2+5) and subtraction (e.g. 6-2) problems as possible during two separate 60 seconds trials. The task consisted of two sheets of paper, an addition and a subtraction version, containing 81 problems presented in three columns. The experimenter used a stopwatch to keep track of time. The number of correctly solved problems was used as the dependent variable.

\section{Arithmetic fact retrieval}


This task was computer administrated and consisted of 12 addition (e.g., 9+5; 4+6) and 12 subtraction problems $(8-4 ; 6-2)$ administered in two separate blocks. One problem at a time was presented horizontally on the computer screen. When the child was ready, the experimenter pressed the mouse button, and a problem was displayed on the screen until the child had responded. A timer started at the onset of the problem and was stopped when the experimenter pressed the mouse button after the child had given an oral response to the problem. The child was instructed to provide an answer immediately by remembering what the answer is and was encouraged to guess if he/she failed to do so. Two different measures were used: 1) number of correctly solved problems with response times within $3 \mathrm{sec}$, 2) number of correctly solved problems including response times longer than $3 \mathrm{~s}$ [77]. Use of finger counting was also registered.

\section{Complex word repetition}

In this verbal working memory task, the child was presented with word sequences. The task was to decide whether each presented word was an animal or not by answering "yes"or "no" (no animal, e.g. car), before the next word was presented. At the end of the sequence, the child had to recall the words in correct (serial) order. The first span size employed was two words, the next was three, and so forth. Two trials were presented for each span size. All children were asked to complete up to span size four, regardless of whether the correct order was recalled. However, if the child managed to recall the correct serial order beyond span size four testing continued until the child failed both trials of the same span length. Half of the words in the sequences were animals. Verbal working memory span was measured as the longest sequence remembered correctly (in serial order), plus 0.5 points if the child managed to recall all trials correctly on the same span size.

\section{Visual matrix task}

A matrix made up of squares was presented some of the squares contained two black dots. The first task was to decide whether these dots were of equal size, and press the "*" key if they were equal or the " $A$ " key if they were not. The child had 3 seconds to respond, after which two additional dots appeared in another square while the former two dots were still visible. The second task was to remember the location of the dots in the matrix. When a sequence of dots has been presented the matrix was removed, and the child was required to draw a cross in the correct squares on an identical empty matrix presented on a sheet of paper. The first matrix had $3 \times 3$ squares and two squares with black dots (i.e., span size two). The next matrix had $3 \times 4$ squares, and three squares with black dots. In this way, the complexity of the matrices increased for each new span size. Testing stopped when the child failed both trials. The same scoring procedure as in the complex word.

\section{Trail-making}

Cognitive shifting was assessed using a paper-and-pencil version of the Trail Making Test [64, 78], composed of two conditions. The first condition (A) consisted of 22 circles, each containing a digit, whereas the second condition's (B) 22 circles contained either a digit or a letter. In condition $A$, the task was to draw a line between the circles in ascending order as quickly as possible. In condition $B$, the children were again told to draw the line and connect the circles in ascending order as fast as possible, but now in alternating order (1-A-2-B-3-C etc.). Seconds needed to complete each condition was used as the dependent measure. Shifting ability was assessed by subtracting the completion time of condition A from B.

\section{Color naming}

This task was administered on two sheets of paper, where 30 "XXX"s (Arial, 22-point font) were printed in different colors (red, green, blue, black and yellow), in two columns. The child was instructed to name the printed color of the XXX's as fast as possible, without making any errors. A stopwatch was used to measure the total response time used as the performance measure. The combined response times for the two sheets of paper were used as a measure of speed of access to semantic information in long-term memory.

\section{Number naming}

The task was administered on two sheets of paper. The singledigit condition consisted of seven rows of the digits 1-9 printed in black ink. Each digit appeared once per row, resulting in 63 . The double-digit condition consisted of six rows and 27 digits, each appearing twice. The participant was told to name each digit as fast as possible, without making any errors. A stopwatch was used to measure the total time needed to name all digits. All children began with the single-digit condition. The combined response time for the single- and double-digit conditions was used as the dependent measure.

\section{Non-symbolic number comparison}

The task was administrated via the Panamath software program (v. 1.21), developed by Halberda and Feigenson [79]. Two arrays were presented, containing between 5-21 blue and yellow dots for $1506 \mathrm{~ms}$. The child had to decide which array was more numerous, and then press the key corresponding to the appropriate side of the screen (F- or L-key). The child had an unlimited amount of time to indicate their response and pressed the space bar to enable the next trial. Prior to each trial, a fixation cross was displayed on the center of the screen. Four ratios (1.24; $1.37 ; 1.60 ; 2.60)$ were presented 26 times each, yielding a total of 104 trials. Two practice trials were performed before the experimental trials. To control for confounding variables, surface area varied on half of the trials, along with dot size. Attention to numerosity was thus ensured. Panamath generated an estimate of ANS acuity (w), based on accuracy at each ratio.

\section{Symbolic number magnitude comparison}

Two digits were simultaneously displayed on the computer screen. The task was to decide, as quickly as possible without making any errors, which of two digits was the numerically larger one. Prior to each problem a "cross" was displayed in the center of the screen for $1000 \mathrm{~ms}$. The child responded by pressing the key corresponding to the appropriate side of the screen. The digits were displayed until the child responded. The test material consisted of one-digit and two-digit numbers that were presented in two separate blocks, starting with the one-digit block. Two 
distances were used, 1 (1-2, 5-6, 8-9, 21-22, 34-33, 74-73, 92-91), and 4-5 (1-6, 3-8, 4-9, 3-7, 31-36, 54-59, 68-63, 97-92) and each digit pair was presented twice (e.g. 2-3 and 3-2) resulting in a total of 32 trials for each block. Response times for correct responses and response accuracy were used as dependent measures.

\section{Subitizing and enumeration}

Arrays of randomly arranged black dots from 1 to 8 , with a diameter of $9 \mathrm{~mm}$, were displayed on the computer screen. The child was instructed to state the number of dots displayed on the screen, as quickly as possible without making any errors. A timer controlled by SuperLAB 4.5 software started at the onset of the problem and was stopped when the experimenter pressed the mouse button after the child had given an oral response to the problem. The screen was empty for $1000 \mathrm{~ms}$ prior to each problem. A total of 24 problems were presented randomly, that is, each number was presented three times. During the task, the experimenter continuously checked the child's answers, registering each error. Response time was used as the dependent measure. Two measures were created $(1-3 ; 5-8)$ to determine subitizing and enumeration range. For each child, a mean response time (correct responses only) was calculated for the two measures.

\section{Time discrimination}

A prospective two-interval time discrimination paradigm was used to measure time perception. The reference stimulus, a red ball, was presented on the center of the computer screen for 3000 ms. After a blank screen was presented for 500 ms, the target stimulus, a blue ball, appeared and remained visible between $1500 \mathrm{~ms}$ to $6000 \mathrm{~ms}$. The task was to determine which of the two stimuli was presented the longest. The child was told to press the corresponding (color-coded) button, the "a"-key was marked with red and "*"-key was marked with blue, after the target stimulus disappeared and was replaced by a response screen. The reference stimulus was fixed at $3000 \mathrm{~ms}$, whereas the target stimulus varied to correspond to four different ratio "bins" $(1: 2,3: 4,4: 5,5: 6)$, across 60 trials. Prior to the task, the child was instructed not to use any counting strategies.

\section{Mental rotation}

The stimuli consisted of alphabetic letters, one letter per item. The test contained 16 items, where the reference was located on the left side accompanied by four comparison stimuli located on the right side adjacent to the target. The comparison stimuli always consisted of two "correct" and two "incorrect" letters. The primary task was to identify the two matching letters, which prompted a mental rotation, and respond by marking them with a pen. Inverted instances of the target (i.e., visually mirrored) were used as incorrect comparison stimuli. All comparison stimuli were rotated only in the picture-plane and in one of six rotation angles $\left(45^{\circ} ; 90^{\circ} ; 135^{\circ} ; 225^{\circ} ; 270^{\circ} ; 315^{\circ}\right)$. The child had to mark both correct comparison stimuli to obtain a point for each item, yielding a maximum score of 16 . The child was allowed 2 minutes to perform the task.

\section{General procedure}

All testing was performed individually over the course of three to four sessions, lasting 30-45 min per session. The total test time for each child was approximately 120-130 minutes. Instructions were given orally, read aloud from a printed manuscript to ensure that every child was given identical information. At least one practice trial was completed for each task following instructions, in order to eliminate misunderstandings. The computeradministered tasks were run on an Apple Power Mac ${ }^{\mathrm{TM}}$ laptop, running SuperLab PRO 4.5 software.

\section{Case Reports}

The performances of the four cases were evaluated in relation to the norm groups by z-scores. A z-score of -1.29 (<10 percentile) or worse was considered to be a substantial deviation from the norm. It should be noted that inferior performance relative to the norm group is always indicated by a negative z-score, even for time measures. Table $\mathbf{2}$ shows the raw scores (z-scores) of the four cases on the general cognitive tasks, the number processing

Table 2 Performance (z-scores) of the four cases of DD on general cognitive processing, number processing, time processing and spatial processing.

\begin{tabular}{|c|c|c|c|c|c|c|}
\hline & C1 & C2 & C3 & C4 & & \\
\hline Task & Boy Grade 2 & Girl Grade 3 & Girl Grade 2 & Boy Grade 2 & Norms Grade 3 & Norms Grade 2 \\
\hline Verbal working memory & $3(-1.13)$ & $3(-1.29)$ & $4(0.32)$ & $2.5(-1.86)$ & $3.98(0.76)$ & $3.78(0.69)$ \\
\hline Visual working memory & $0(-4.22)$ & $0(-3.02)$ & $2(-1.65)$ & $2.5(-1.01)$ & $3.36(1.11)$ & $3.29(0.78)$ \\
\hline Shifting (trail-making) & $111(0.02)$ & $193(-2.71)$ & $270(-2.87)$ & $214(-1.85)$ & $71(45)$ & $112(55)$ \\
\hline Color naming & $58(0.14)$ & $68(-0.93)$ & $67(-0.49)$ & $58(0.14)$ & 55 (13.95) & $60(14.33)$ \\
\hline Number naming & $194(-0.22)$ & $96(0.34)$ & $174(0.18)$ & $90(1.90)$ & $107(32)$ & $183(49)$ \\
\hline NSND (Weber fraction) & $0.61 * *(-4.44)$ & $0.72(-5.67)$ & $0.29(-0.87)$ & $0.22(-0.11)$ & $0.21(0.09)$ & \\
\hline 1-digit comparison (RT) & $1.10(0.63)$ & $1.84(-1.76)$ & $0.99(1.00)$ & $0.95(1.13)$ & $0.96(0.50)$ & $1.29(0.30)$ \\
\hline 1-digit accuracy & $27(-1.47)$ & $32(0.97)$ & $26(-1.99)$ & $30(0.09)$ & $30.24(1.81)$ & $29.83(1.92)$ \\
\hline 2-digit comparison (RT) & $1.67(0.52)$ & $2.70(-3.29)$ & $1.55(0.73)$ & $1.77(0.34)$ & $1.35(0.41)$ & $1.96(0.56)$ \\
\hline 2-digit accuracy & $28(0.26)$ & $26(-2.19)$ & $25(-1.24)$ & $30(0.09)$ & $30.33(1.98)$ & $28.80(3.06)$ \\
\hline Subitizing 1-3 & $1.42(-0.88)$ & $1.58(-1.00)$ & $1.65(-1.83)$ & $1.14(0.29)$ & $1.26(0.32)$ & $1.21(0.24)$ \\
\hline Enumeration 5-8 & $3.40(0.55)$ & $6.11(-3.79)$ & $5.03(-2.03)$ & $4.28(-0.87)$ & $3.38(0.72)$ & $3.74(0.62)$ \\
\hline Time discrimination & $25(-2.64)$ & $21(-3.31)$ & $38(-0.57)$ & $42(0.07)$ & $43.44(6.78)$ & $41.58(6.28)$ \\
\hline Mental rotation & $2(-1.47)$ & $5(-1.47)$ & $10(0.44)$ & $9(0.20)$ & $10.76(3.92)$ & $8.16(4.20)$ \\
\hline
\end{tabular}

NSND=Non-Symbolic Number Discrimination

**Norms from the Panamath test software for eight year olds $10^{\text {th }}$ percentile: Weber fraction $=0.57$ 
tasks, the time processing task and the spatial processing task along with descriptive statistics for the norm groups.

\section{Assessment of general cognitive abilities}

As indicated by the z-scores, C1 had an impaired visuospatial working memory $(z=-4.22)$, whereas his verbal working memory was within the normal range $(z=-1.13)$. He also performed within the normal range on the trail-making task $(z=-0.02)$, tapping the executive function of shifting between retrieval strategies or operations. He displayed normal processing speed on the color naming and number naming tasks $(z=0.14 ;-0.22)$.

C2's z-scores suggest that she had impaired verbal working memory ( $z=-1.29)$, visuospatial working memory $(z=-3.02)$, and a defective shifting ability $(z=2.71)$, whereas her general processing speed, tapped by the color naming task $(z=-0.93)$ and the number naming task $(z=0.34)$, was within normal range.

C3's z-scores indicated deficits in visuospatial working memory ( $z=-1.65)$ and (executive) shifting ability $(z=-2.87)$. On the other hand, her verbal working memory $(z=0.32)$, and general processing speed, tapped by the color and number naming tasks ( $z=-.049 ; z=0.18)$, were intact.

C4 showed poor verbal working memory capacity $(z=-1.86)$ and defective shifting ability ( $z=-1.85)$. In contrast, his visuospatial working memory capacity $(z=-1.01)$ and general processing speed, tapped by the color naming task $(z=0.14)$ and the number naming task $(z=1.90)$, were within the normal range.

\section{Assessment of number, time, and spatial processing}

C1's w-score ( $z=-4.44)$ for the non-symbolic number discrimination task suggest a deficit in the ANS. Except for a low accuracy score on the single-digit comparison task $(z=-1.47)$, C1 performed within the normal range on all other number processing tasks. C1 also performed poorly on the time discrimination task ( $z=$ $2.64)$ and the mental rotation task ( $z=-1.47)$, indicating impaired temporal and spatial processing.

Similar to C1, C2 displayed severe problems with the non-symbolic number discrimination task $(z=-5.67)$, the time discrimination task $(z=-3.31)$ and the mental rotation task $(z=-1.47)$. C2 also performed poorly on the RT measure of the single-digit comparison task $(z=-1.76)$ and the two measures of the doubledigit comparison task (RT: $z=-3.29$; accuracy: $z=-2.19$ ). In addition, C2's ability to enumerate $5-8$ dots quickly was impaired ( $z=-3.79$ ), but not the ability to subitize $1-3$ dots $(z=-1.00)$. C2's performance pattern showed an overall number processing deficit and an impaired ability to process temporal and spatial information.

C3 obtained low accuracy scores on the single-digit comparison task ( $z=-1.99)$, the double-digit comparison task $(z=-1.24)$ and displayed slow performance on the subitizing $(z=-1.83)$ and enumeration ( $z=-2.03)$ measures. In contrast, C3's performance on the non-symbolic number discrimination task ( $z=-0.87)$ and the time discrimination task $(z=-0.57)$ and the mental rotation task $(z=0.44)$ were within the normal range. The presentation format of the subitzing task and enumeration task is non-symbolic, but responses prompt a vocal response in the form of number words.
Exhibiting impaired performance on these tasks while showing intact performance on number discrimination together indicates that her difficulties were primarily in symbolic processing.

C4 displayed normal performance on all number processing tasks. Furthermore, his performance on the time discrimination task $(z=0.07)$ and the mental rotation task $(z=0.20)$ were within normal range. Thus, $\mathrm{C} 4$ did not appear to have any deficiencies related to the processing of numerical, spatial and temporal information.

\section{Discussion}

The aim of the present study was to further understand the heterogeneity of $D D$, by testing the main core deficit accounts in relation to four cases of DD with similar profiles of mathematical deficits. The ATOM model, that a general magnitude-processing deficit underlies DD, was also tested. The question was whether all four cases displayed similar cognitive deficit profiles consistent with the same or different core deficit accounts. The latter outcome would suggest that DD originates from multiple deficits. The four cases will now be discussed in relation to the different accounts.

\section{C1 - Magnitude processing subtype}

The deficit profile of $\mathrm{C} 1$ entails impaired non-symbolic number processing [11, 14-16, 23, 80], and impaired temporal and spatial processing. These deficits indicate that he has not only a defective ANS, but also a general magnitude processing deficit, as previously reported by Skagerlund and Träff [27] in a group of fourth graders with DD, see also [25, 26, 28]. C1's profile also encompasses a defective visuospatial working memory capacity, cf. [57, 62]. Given that C1 showed normal shifting ability and verbal working-memory, the apparent deficit in visuospatial working-memory is likely caused by a general magnitude processing deficit, consistent with the ATOM model. Although working-memory functionality in general may be intact in $\mathrm{C} 1$, he may have problems in encoding and retaining spatial information in working-memory. Visuospatial working memory processing involves the right fronto-parietal network, comprising of the right IPS and the right inferior frontal gyrus [81]. The inferior frontal gyrus may in this case be intact, but receives impoverished spatial information from the IPS because of a magnitude processing deficit.

Previous hypotheses have stated that due to shared neural resources between quantity representations and other continuous magnitude processes, a deficit in quantity processing should affect other dimensions as well, such as time and space [43]. Time perception has been attributed to neural processing in the parietal cortex and insula $[82,83]$, whereas Kucian et al. [84] found that mental rotation tasks are subserved by cortical substrates in the IPS. The intraparietal cortex is also known to be involved in visual attention [85], which could explain the concomitant and severe visuospatial working-memory deficit shown by $\mathrm{C} 1$. Visual attention may project information via dorsal visual stream to frontal areas to be encoded in working-memory, in this case set of dots. As C1 did not show impairments in verbal working memory, one tentative interpretation is that the deficit is 
limited to visuospatial information sub-served by occipitoparietal visual processing. The posterior IPS has structural connections with extrastriate visual areas [86], indicating that this cortical circuitry may be susceptible to abnormalities. They could subsequently hamper visuospatial processing. Uddin et al. [86] suggested that the posterior IPS may play a role in transforming symbolic and non-symbolic numerical information to spatial and semantic representations of quantity. Additionally, the anterior IPS has structural connections with insula [86], which is involved in time perception [50] and might be part of a structural network for magnitude processing in general.

In sum, the cognitive profile of $\mathrm{C} 1$ suggests primary DD with a magnitude processing deficit that impedes mathematical reasoning. Neurocognitive correlates would likely be traced to the IPS and the dorsal visual stream.

\section{C2 - Magnitude processing and general subtype}

C2 has a complex deficit profile; a general number processing deficit that involved non-symbolic and symbolic number processing and enumeration ability, but intact subitizing ability. This pattern is consistent with the defective ANS account, e.g., $[15,16,23]$. Another interesting aspect of C2's profile is the impaired ability to process temporal and spatial information, like $\mathrm{C} 1$, which in combination with her number processing deficits suggest a general magnitude processing deficit. C2's deficit profile also points to general cognitive deficits, such as impaired verbal and visuospatial working memory [62] and a defective executive function of shifting $[64,78]$.

Given C2's widespread cognitive impairments, it is hard to point to any singular cortical area that might be dysfunctional. However, it is likely that a few key structural connections or cortical loci are dysfunctional, which in turn cascade into largescale cognitive deficits of number processing as well as domaingeneral processing. Nevertheless, it is likely that $C 2$ has the same cortical deficits as $\mathrm{C} 1$, with additional deficits pertaining to frontal areas of the brain.

In sum, C2 likely suffers from primary DD characterized by a combined magnitude processing deficit and general cognitive impairment.

\section{C3 - Access deficit and general subtype}

C3's deficit profile is characterized by defective symbolic number processing, but intact non-symbolic number processing, cf. [50, 51]. Thus, consistent with the access deficit hypothesis and possibly the defective OTS hypothesis, as C3's subitizing ability was impaired [44, 46, 47]. Her deficit profile also involves deficits in visuospatial working memory and (executive) shifting ability. C3 highlights the likely dissociation between nonsymbolic approximate number processing (taxing the ANS), exact enumeration and subitizing. The results indicate that the magnitude processing system is intact overall, as C3 had an unimpaired ANS and normal processing of time and space.

In contrast, C3 displayed problems with several tasks relying heavily on neurocognitive processing and neural loci in the frontal areas of the brain. Visuospatial working memory processing involves a right fronto-parietal network, comprising of the right IPS and the right inferior frontal gyrus. As C3 showed an unimpaired ANS, which relies on IPS bilaterally, one interpretation is that either (a) the cortical dysfunction is circumscribed to inferior frontal gyrus or (b) a white matter connectivity issue impedes information processing in the fronto-parietal network. Support for the latter comes from the impaired performance on the symbolic number processing task. Although C3 had no problems with rapidly naming digits, she did struggle with selecting which number is the highest in the symbolic number comparison task. This suggests that C3 suffers from an access deficit that impedes the accurate mapping of symbols to their underlying quantity representation. The underlying neural dysfunction responsible for the access deficit is unclear. C3 could rapidly decode digits and express them verbally, which may indicate that the ability to process complex visual stimuli sub-served by the fusiform gyrus, lingual gyrus and hippocampal regions is intact. One tentative interpretation is that frontal regions, such as dorsolateral prefrontal cortex and inferior frontal gyrus, is implicated during access to the semantic referents [87], and may be involved during active counting due to effortful working memory demands.

Throughout ontogenetic development, typically developing children show increased white matter density in the frontal lobes, suggesting a maturation of the fronto-parietal network [53]. Children with DD, however, do not show the same significant increase in white matter volume, which may indicate that DD may be partly due to rudimentary associations between symbols and their underlying magnitude representations [54]. Given that C3 also show extensive deficits in working-memory capacity and shifting ability, partly relying on the prefrontal cortex, it is likely that frontal dysfunctions inhibit active and explicit processing of numerical content. This would also explain C3's apparent difficulty with subitizing and enumeration, since the task requires verbal answers.

Taken together, C3 may have primary DD with an access deficit subtype conjointly with a general cognitive impairment.

\section{C4 - Domain-general subtype}

The deficit profile of $\mathrm{C} 4$ is restricted to poor verbal working memory, cf. [62], and defective shifting ability, cf. [47, 64]. Thus, corresponding with the general cognitive deficit account of DD. Shifting ability and verbal working-memory rely heavily on the cognitive control network or salience network [88, 89], comprising of anterior cingulate cortex (ACC), insula, and dorsolateral prefrontal cortex. Given that insula and ACC are important in magnitude processing as well, especially during time processing, these areas are likely intact in $\mathrm{C} 4$ due to the normal magnitude processing capabilities. Thus, C4 may have problems in encoding and retaining verbal information in working-memory during mathematical reasoning, which may hamper the ability to maintain control of intermediate steps during calculation and problem-solving.

In contrast to the previous cases, C4 cannot be characterized as having primary DD, as the mathematical difficulties are likely caused by a domain-general cognitive impairment; hence, C4 likely has secondary DD [3]. 


\section{Concluding Discussion}

The present study clearly demonstrates that only relying on traditional studies utilizing group comparisons is not sufficient for exploring the heterogeneity of DD. Collapsing all individuals into one, supposedly, homogeneous group may obfuscate important inter-individual variability. The different cognitive deficit profiles of the present cases provide strong support for a multideficits account of $\mathrm{DD}$, further corroborating findings reported by Bartelet et al. [69], Iuculano et al. [71], and Skagerlund and Träff [5]. Furthermore, not only did the cases display profiles consistent with different accounts, three of the cases displayed profiles consistent with more than one account [46]. The profiles of cases C1 and C2 are consistent with the ANS deficit hypothesis, the general deficit account and, interestingly, the general magnitudeprocessing deficit account.

C3 had an access deficit in combination with a general cognitive deficit. C4, however, only suffered from general cognitive deficits. These patterns of deficits are important as they indicate that DD can originate from different constellations of deficits at an individual level. This is important to consider when planning and executing interventions, because it might be necessary to target several different cognitive skills.

Regarding the different core deficit accounts, it is somewhat unexpected to find that all four cases performed consistently with the general cognitive account. More specifically, all cases have either verbal and/or visuospatial working memory deficits [62]. In addition, all cases except $\mathrm{C} 1$, display executive function impairment in shifting between retrieval strategies or operations $[64,78]$. Thus, none of the cases show a deficit circumscribed to number processing alone, which is the defining feature of DD according to some researchers [4]. In contrast, general cognitive deficits are identified in one case, $\mathrm{C} 4$.

Although promising, the distinction between primary and secondary DD proved to be not entirely unequivocal. Kaufmann et al. [3] proposed that secondary DD should be used if "numerical/arithmetic dysfunctions are entirely caused by nonnumerical impairments (e.g., attention disorders)" (p. 4). We agree with the authors and believe that the definition is sound, but it proved hard to disentangle the domain-general processes from numerical processes even at an individual level. For example, C3 displayed a profile suggestive of an access deficit. However, given the coexisting deficits in executive functions and visuospatial working-memory, it cannot be ruled out that these deficits play a causative role in the apparent number processing deficit. Executive functions may have affected performance on the symbolic number comparison task during the task situation itself, or executive functions may have hampered the mapping between symbols over the long-term throughout development. By factoring in that $\mathrm{C} 3$ has normal reading skills, involving symbols and their semantic referents, and performed normally on the non-symbolic ANS task involving selection of either of two alternatives, it is plausible that C3 has a "genuine" deficit in number processing involving symbols. Nevertheless, it is unclear whether $\mathrm{C} 3$ can be said to suffer from an access deficit and fit the profile of Rousselle and Noël [50], given the concomitant domain- general deficits. However, it is likely that the mathematical difficulties of C3 cannot be attributed entirely too non-numerical factors, which is the defining feature of secondary DD. That brings us to whether C3 suffers from primary DD, which is defined as a "heterogeneous disorder resulting from individual deficits in numerical or arithmetic functioning at behavioral, cognitive/ neuropsychological and neuronal levels" [3]. The access deficit shown by C3 fits this definition nicely, but it is unclear to what extent domain-general abilities can contribute to these defining characteristics. One strict interpretation of primary DD would be that one has to show only number processing deficits while displaying no domain-general deficits whatsoever. A more lenient interpretation would be that a number processing deficit is both a necessary and sufficient criterion of primary DD, in which case number processing deficits and domain-general deficits can coexist. This ambiguity led us to interpret C3 as having primary DD with an access deficit subtype with concurrent domaingeneral subtype. We welcome the distinction between primary DD and secondary DD, but we urge for further discussion about the heterogeneity and the defining features of each of these. Further work should also address the challenges in disentangling different cognitive processes and deficits when assessing children at risk of developing DD so that appropriate interventions can be implemented.

A key finding is that two of the cases $(\mathrm{C} 1, \mathrm{C} 2)$ displayed a general magnitude-processing deficit. In view of the ATOM model [29, 30], the deficit profiles of $C 1, C 2$ and $C 3$ suggest that children with DD suffering from a deficit in the innate ANS $(\mathrm{C} 1 ; \mathrm{C} 2)$ might also have impaired spatial and temporal processing skills. They should therefore be regarded as having a general magnitude processing deficit. The child that showed an access deficit, C3, however showed impaired symbolic number processing but intact magnitude processing skills in all dimensions. This dissociation suggests that symbolic number processing is connected to dimension-specific number processes and not to the partly shared general magnitude system.

Using a comprehensive test battery to investigate the cognitive profiles in depth, we can make nuanced interpretations about specific weaknesses. For example, C1 showed weakness of the ANS, magnitude processing and visuospatial-working memory capacity, consistent with two prominent hypotheses about DD. One advantage of our methodological approach is that we can triangulate cognitive weaknesses. C1 showed impaired visuospatial working memory capacity, and if a traditional group analysis with a single specific hypothesis about the importance of visuospatial working memory ability had been used, a quite heterogeneous group showing impaired visuospatial workingmemory processing might have been found. However, the individuals in this group might have displayed this impairment for different underlying reasons. Some individuals might have genuine neurocognitive issues pertaining to deficient activity in frontal areas of the brain, whereas others - such as C1 - have a dysfunction in a precursor process in IPS involving magnitude information. The dysfunctional magnitude process may then propagate impoverished information via dorsal stream to frontal areas of the brain responsible for subsequent workingmemory processes. By administering a comprehensive set of 
tasks, we tentatively suggest that the apparent difficulties in visuospatial working memory displayed by $\mathrm{C} 1$ is a by-product of another process, hence getting a more nuanced and elaborate understanding of the entire cognitive profile of an individual with DD. Although making matters more complex, and naturally subject to both replication and verification using imaging data such as fMRI and DTI, we want to show that DD is a complex condition and we should treat it as such. This requires a multilevel approach, and we cannot rely solely on group analyses that measure single constructs collapsed across numerous individuals at a single point in time. Likewise, our findings from this case study also warrant further investigations to verify these findings, and also to ameliorate the inherent limitations in generalizability of this case study design. We acknowledge the tentative nature of our neurocognitive hypotheses, but we hope that these can inform and guide future neuroimaging studies that broaden our understanding of this very complex learning disorder.

\section{Acknowledgement}

This research was supported by grants from the Swedish Research Council for Health, Working Life and Welfare (2008-0238 and 2010-0078) awarded to Ulf Träff. 


\section{References}

1 American Psychiatric Association (1994) Diagnostic and Statistical Manual of Mental Disorders, DSM-IV, American Psychiatric Association, Washington, DC.

2 GearyDC(1993)Mathematical disabilities: cognitive, neuropsychological, and genetic components. Psychol Bull 114: 345-362.

3 Kaufmann L, Mazzocco MM, Dowker A, von Aster M, Göbel SM, et al. (2013) Dyscalculia from a developmental and differential perspective. Front in Dev Psychol 4: 516.

4 Rubinsten O, Henik A (2009) Developmental Dyscalculia: heterogeneity might not mean different mechanisms. Trend Cogn Sci 13: 92-99.

5 Skagerlund K, Träff U (2014) Number processing and heterogeneity of developmental dyscalculia: subtypes with different cognitive profiles and deficits. J Learn Dis 10: 1177.

6 Vicario CM, Rappo G, Pepi A, Pavan A, Martino D, et al. (2012) Temporal Abnormalities in Children With Developmental Dyscalculia. Dev Neuropsychol 37: 636-652.

7 Price GR, Ansari D (2013) Dyscalculia: Characteristics, Causes, and Treatments. Numeracy 6.

8 Dehaene S (2011) The number sense. New York: Oxford University Press.

9 Gelman R, Butterworth B (2005) Number and language: How are they related? Trend Cogn Sci 9: 6-10.

10 Piazza M (2010) Neurocognitive start-up tools for symbolic number representations. Trend Cogn Sci 14: 542-551.

11 Mazzocco MMM, Feigenson L, Halberda J (2011) Impaired Acuity of the Approximate Number System Underlies Mathematical Learning Disability (Dyscalculia). Child Dev 82: 1224-1237.

12 Gilmore CK, McCarthy SE, Spelke ES (2010) Non-symbolic arithmetic abilities and mathematics achievement in the first year of formal schooling. Cognition 115: 394-406.

13 Mazzocco MMM, Feigenson L, Halberda J (2011) Preschoolers' precision of the Approximate Number System predicts later school mathematics performance. PLoS ONE 6: e23749.

14 Landerl K, Fussenegger B, Moll K, Willburger E (2009) Dyslexia and dyscalculia: Two learning disorders with different cognitive profiles. J Exp Child Psychol 103: 309-324.

15 Mejias S, Mussolin C, Rousselle L, Grégoire J, Noël M-P, et al. (2012) Numerical and non-numerical estimation in children with and without mathematical learning disabilities, Child Neuropsychol 18: 550-575.

16 Piazza M, Facoetti A, Trussardi AN, Berteletti I, Conte S, Lucangel D, Dehaene S, Zorzi M, et al. (2010) Developmental trajectory of number acuity reveals a severe impairment in developmental dyscalculia. Cognition 116: 33-41.

17 Butterworth B (2010) Foundational numerical capacities and the origins of dyscalculia. Trend Cogn Sci 14: 534-541.

18 Zorzi M, Stoianov I, Umilta C (2005) Computational modelling of numerical cognition. Campbell JID (Ed.), Handbook of mathematical cognition (pp. 67-84). New York: Psychology Press.

19 Chan WWL, Au TK, Tang J (2013) Developmental dyscalculia and low numeracy in Chinese children. Res in Dev Disabil, 34: 1613-1622.
20 Gruber O, Indefrey P, Steinmetz H, Kleinschmidt A (2001) Dissociating neural correlates of cognitive components in mental calculation. Cerebral Cortex 11: 350-359.

21 Ashkenazi S, Rosenberg-Lee M, Tenison C, Menon V (2012) Weak taskrelated modulation and stimulus representations during arithmetic problem solving in children with developmental dyscalculia. Dev Cogn Neurosci 2S: 152-166.

22 Kaufmann L, Wood G, Rubinsten O, Henik A (2011) Meta-analyses of developmental $\mathrm{fMRI}$ studies investigating typical and atypical trajectories of number processing and calculation. Dev Neuropsych 36: 763-787.

23 Price GR, Holloway I, Räsänen $P$, Vesterinen $M$, Ansari $D$, et al. (2007) Impaired parietal magnitude processing in developmental dyscalculia. Curr Biol 17: R1042-R1043.

24 luculano T, Cohen Kadosh R (2014) Preliminary evidence for performance enhancement following parietal lobe stimulation in Developmental Dyscalculia. Front in Hum Neurosci 8: 38.

25 Ashkenazi S, Henik A (2010) A disassociation between physical and mental number bisection in developmental dyscalculia. Neuropsychologia 48: 2861-2868.

26 Moll K, Göbel SM, Gooch D, Landerl K, Snowling MJ (2014) Cognitive Risk Factors for Specific Learning Disorder: Processing Speed, Temporal Processing, and Working Memory. J Learn Dis 10: 1177.

27 Skagerlund K, Träff U (2014) Development of magnitude processing in children with developmental dyscalculia: space, time, and number. Front in Psychol 5: 675.

28 Vicario CM, Pecoraro P, Turriziani P, Koch G, Caltagirone C, Oliveri M, et al. (2008) Relativistic compression and expansion of experiential time in the left and right space. PLoS One 3: e1716.

29 Bueti D, Walsh V (2009) The parietal cortex and the representation of time, space, number and other magnitudes. Phil Trans Royal Soc Biol Sci 364: 1831-1840.

30 Walsh V (2003) A theory of magnitude: common cortical metrics of time, space and quantity. Trend Cogn Sci 7: 483-488.

31 Cantlon JF, Platt ML, Brannon EM (2009) Beyond the number domain. Trend Cogn Sci 13: 83-91.

32 Cappelletti M, Freeman ED, Cipolotti L (2009) Dissociations and interactions between time, numerosity and space processing. Neuropsychologia 47: 2732-2748.

33 Dehaene S, Bossini S, Giraux P (1993) The mental representation of parity and number magnitude. J Exp Psychol Gen 122: 371-396.

34 Moyer RS, Landauer TK (1967) Time required for judgment of numerical inequality. Nature 215: 1519-1520.

35 Fulbright RK, Manson SC, Skudlarski P, Lacadie CM, Gore JC, et al. (2003) Quantity determination and the distance effect with letters, numbers, and shapes: a functional MR imaging study of number processing. Am J Neuroradiology 23: 193-200.

36 Dormal V, Seron X, Pesenti M (2006) Numerosity-duration interference: a Stroop experiment. Acta Psychologica 121: 109-124.

37 Rusconi E, Kwan B, Giordano BL, Umilta C, Butterworth B, et al. (2006) Spatial representation of pitch height: the SMARC effect. Cognition 99: 113-129.

38 Fias W, Lammertyn J, Reynvoet B, Dupont P, Orban GA, et al. (2003) Parietal representation of symbolic and nonsymbolic magnitude. J Cogn Neurosci 15: 1-11. 
39 Ishihara M, Keller PE, Rossetti Y, Prinz W (2008) Horizontal spatia representation of time: Evidence for the STEARC effect. Cortex 44: 454-461.

40 Chang AYC, Tzeng OJL, Hung DL, Wu DH (2011) Big time is not always long: numerical magnitude automatically affects time reproduction. Psychol Sci 22: 1567-1573.

41 Cappelletti M, Freeman ED, Cipolotti L (2011) Numbers and time doubly dissociate. Neuropsychologia 49: 3078-3092.

42 Fabbri MJ, Cancellieri J, Natale V (2012) The A Theory of Magnitude (ATOM) model in temporal perception and reproduction tasks. Acta Psychologica 139: 111-123.

43 Feigenson L (2007) The equality of quantity. Trend Cogn Sci 11: 185-187.

44 Desoete A, Grégoire J (2006) Numerical competence in young children and in children with mathematics learning disabilities. Learn Indiv Diff 16: 351-367.

45 Wilson AJ, Dehaene S (2007) Number sense and developmental dyscalculia. Coch D, Dawson G, Fischer K (Eds.), Human behavior, learning, and the developing brain: Atypical development (pp. 212238). New York: Guilford.

46 Andersson U, Östergren R (2012) Number magnitude processing and basic cognitive functions in children with mathematical learning disabilities. Learn Indiv Diff 22: 701-714.

47 Moeller K, Neuburger S, Kaufmann K, Landerl K, Nuerk HC, et al. (2009) Basic number processing deficits in developmental dyscalculia: Evidence from eye tracking. Cogn Dev 24: 371-386.

48 Furman T, Rubinsten O (2012) Symbolic and non-symbolic numerica representation in adults with and without developmental dyscalculia. Behav Brain Funct 8: 1-15.

49 Ashkenazi S, Mark-Zigdon N, Henik A (2013) Do subitizing deficits in developmental dyscalculia involve pattern recognition weakness? Dev Sci 16: 35-46.

50 Rousselle L, Noël M-P (2007) Basic numerical skills in children with mathematics learning disabilities: A comparison of symbolic vs nonsymbolic number magnitude processing. Cognition 102: 361-395.

51 De Smedt B, Gilmore CK (2011) Defective number module or impaired access? Numerical magnitude processing in first graders with mathematical difficulties. J Exp Child Psychol 108: 278-292.

52 Landerl K, Kölle C (2009) Typical and atypical development of basic numerical skills in elementary school. J Exp Child Psychol 103: 546-565.

53 Ranpura A, Isaacs E, Edmonds C, Rogers M, Lanigan J, et al. (2013) Developmental trajectories of grey and white matter in dyscalculia. Trend Neurosci Edu 2: 56-64.

54 Rykhlevskaia E, Uddin LQ, Kondos L, Menon V (2009) Neuroanatomical correlates of developmental dyscalculia: combined evidence from morphometry and tractography. Front Hum Neurosci 3

55 Kucian K, Schwizer S, Ashkenazi S, Hänggi J, Rotzer S, et al.(2014) Developmental dyscalculia: a dysconnection syndrome. Brain Struct Funct 219: 1721-1733.

56 Geary D, Hoard M (2005) Learning disabilities in arithmetic and mathematics: Theoretical and empirical perspectives. Campbell JID (Ed.), Handbook of mathematical cognition (pp. 253-267). New York, NY: Psychology Press.

57 Andersson U (2010) Skill development in different components of arithmetic and basic cognitive functions: Findings from a threeyear longitudinal study of children with different types of learning difficulties. J Edu Psychol 102: 115-134.

58 Andersson U, Lyxell B (2007) Working Memory deficits in children with mathematical difficulties: A General or Specific Deficit? J Exp Child Psychol 96: 197-228.

59 Bull R, Johnston R, Roy JA (1999) Exploring the roles of the visualspatial sketchpad and central executive in children's arithmetical skills: Views from cognition and developmental neuropsychology. Dev Neuropsychol 15: 421-442.

60 D'Amico A, Passolunghi MC (2009) Naming speed and effortful and automatic inhibition in children with arithmetic learning disabilities. Learn Indiv Diff 19: 170-180.

61 Passolunghi MC, Cornoldi C (2008) Working memory failures in children with arithmetical difficulties. Child Neuropsychol 14: 387-400.

62 Raghubar KP, Barnes MA, Hecht SA (2010) Working memory and mathematics: A review of developmental, individual difference, and cognitive approaches. Learn Indiv Diff 20: 110-122.

63 Swanson HL, Beebe-Frankenberger M (2004) The relationship between working memory and mathematical problem solving in children at risk and not at risk for serious math difficulties. J Edu Psychol 96: 471-491.

64 van der Sluis S, de Jong PF, van der Leij A (2004) Inhibition and shifting in children with learning deficits in arithmetic and reading. J Exp Child Psychol 87: 239-266.

65 Dowker A (2005) Individual differences in arithmetic: Implications for psychology, neuroscience and education. Hove, East Sussex: Psychology Press.

66 Jordan NC, Kaplan D, Hanich LB (2002) Achievement growth in children with learning difficulties in mathematics: Findings of a twoyear longitudinal study. J Edu Psychol 94: 586-597.

67 Kosc L (1974) Developmental dyscalculia. J Learn Dis 7: 165-171.

68 Mazzocco MMM, Myers GF (2003) Complexities in identifying and defining mathematics learning disability in the primary school years. Ann Dyslexia 53: 218-253.

69 Bartelet D, Ansari D, Vaessen A, Blomert L (2014) Cognitive subtypes of mathematics learning difficulties in primary education. Res Dev Dis 35: 657-670.

70 von Aster M, Shalev R (2007) Number development and developmental dyscalculia. Dev Med Child Neurol 49: 868-873.

71 Iuculano T, Tang J, Hall CWB, Butterworth B (2008) Core information processing deficits in developmental dyscalculia and low numeracy. Dev Sci 11: 669-680.

72 De Visscher A, Noël MP (2013) A case study of arithmetic facts dyscalculia caused by a hypersensitivity-to-interference in memory. Cortex 49: 50-70.

73 Raven JC (1976) Standard progressive matrices. Oxford: Oxford Psychologists Press.

74 Wechsler D (2003) Wechsler Intelligence Scale for Children-Fourth Edition (WISC-IV) administration and scoring manual. San Antonio, TX: The Psychological Corporation.

75 Elwér Å, Fridolfsson I, Samuelsson S, Wiklund C (2009) LäSt Handledning - Test i läsning- och stavning för åk 1 - 5. Linköping: VasaTryck AB.

76 Malmquist E (1977) Reading and writing difficulties in children Analysis and treatment. Lund: Gleerups. 
77 Russell RL, Ginsburg HP (1984) Cognitive analysis of children's mathematical difficulties. Cogn Instruct 1: 217-244.

78 McLean JF, Hitch GJ (1999) Working memory impairments in children with specific arithmetic learning diffculties. J Exp Child Psychol 74: 240-260.

79 Halberda J, Feigenson L (2008) Developmental change in the acuity of the 'Number Sense': The Approximate Number System in 3-, 4-, 5-, and 6-year-olds and adults. Dev Psychol 44: 1457-1465.

80 Mussolin C, Mejias S, Noël M-P (2010) Symbolic and non-symbolic number comparison in children with and without dyscalculia. Cognition 115: 10-25.

81 Rotzer S, Loenneker T, Kucian K, Martin E, Klaver P, von Aster M, et al. (2009) Dysfunctional neural network of spatial working memory contributes to developmental dyscalculia. Neuropsychologia 47: 2859-2865.

82 Lewis PA, Miall RC (2003) Brain activation patterns during measurement of sub- and supra-second intervals. Neuropsychologia 41: 1583-1592.

83 Wiener M, Turkeltaub P, Coslett HB (2010) The image of time: A voxel-wise meta-analysis. Neurolmage 49: 1728-1740.
84 Kucian K, von Aster M, Loenneker T, Dietrich T, Mast FW, et al. (2007) Brain activation during mental rotation in school children and adults. J Neur Transm 114: 675-686

85 Klingberg T, Forssberg $\mathrm{H}$, Westerberg $\mathrm{H}$ (2002) Increased brain activity in frontal and parietal cortex underlies the development of visuospatial working memory capacity during childhood. J Cogn Neurosci 14: 1-10.

86 Uddin LQ, Supekar K, Amin H, Ryklevskaia E, Nguyen DA, et al. (2010) Dissociable Connectivity within Human Angular Gyrus and Intraparietal Sulcus: Evidence from Functional and Structural Connectivity. Cerebral Cortex 20: 2636-2646.

87 Nieder A (2009) Prefrontal cortex and the evolution of symbolic reference. Curr Op Neurobiol 19: 99-108.

88 Engström M, Landtblom A-M, Karlsson T (2013) Brain and effort: brain activation and effort-related working memory in healthy participants and patients with working memory deficits. Front Hum Neurosci, 7: 140.

89 Menon V, Uddin LQ (2010) Saliency, switching, attention, and control: a network model of insula function. Brain Struct Funct, 214: 655-667. 